\title{
Alterations of Asymmetric Dimethylarginine (ADMA)-Containing Protein Profiles Associated with Chronic Pancreatitis Pathogenesis
}

\author{
Chaochao Tan' \\ Yan Xiao ${ }^{2}$ \\ Xiangping Huang' \\ Ling $\mathrm{Wu}^{\prime}$ \\ Ying Huang ${ }^{3}$ \\ 'Department of Clinical Laboratory, \\ Hunan Provincial People's Hospital \\ (The First Affiliated Hospital of Hunan \\ Normal University), Changsha, People's \\ Republic of China; ${ }^{2}$ Intensive Care \\ Unit, Hunan Provincial People's \\ Hospital (The First Affiliated Hospital \\ of Hunan Normal University), \\ Changsha, People's Republic of China; \\ ${ }^{3}$ Department of Emergency, Hunan \\ Provincial People's Hospital (The First \\ Affiliated Hospital of Hunan Normal \\ University), Changsha, People's \\ Republic of China
}

Objective: The pathophysiological mechanisms of chronic pancreatitis (CP) still remain poorly understood. In this study, we aimed to characterize asymmetric dimethylarginine (ADMA)-containing proteins in pancreatic tissues and its relationship with CP pathogenesis. Methods: Totally 36 patients with CP were enrolled in this study. Seven other cholangiocarcinoma patients without pancreas involvements or patients with benign pancreatic tumors were included as the control group. Total proteins in human pancreatic tissues were digested by trypsin, and ADMA-containing peptides were enriched via immunoaffinity purification. The LC-MS/MS was performed to characterize ADMA-containing peptides and their modification sites in CP tissues. Relative asymmetric arginine dimethylation levels of HNRNPA3 proteins in human pancreatic tissues were detected by the immunoprecipitation combined with Western blot. The serum inflammatory factors were determined via the ELISA method. Results: A total of 134 ADMA sites in the control group and 137 ADMA sites in CP tissues were characterized by mass spectrometry, which belong to 93 and 94 ADMA-containing proteins in the control group and CP tissues, respectively. Glycine and proline residues were significantly overrepresented in the flanking sequences of ADMA sites. ADMA-containing proteins in the $\mathrm{CP}$ tissues were associated with various biological processes, especially the RNA metabolism and splicing pathways. Multiple protein members of the spliceosome pathway such as HNRNPA3 possess ADMA sites in the CP tissues. HNRNPA3 dimethylation levels were greatly increased in $\mathrm{CP}$ tissues, which were positively correlated with inflammatory factors.

Conclusion: The pathogenesis of $\mathrm{CP}$ is associated with alterations of asymmetric arginine dimethylation in pancreatic tissues.

Keywords: chronic pancreatitis, arginine methylation, ADMA, proteomics, HNRNPA3

\section{Introduction}

Chronic pancreatitis (CP) is commonly defined as the fibroinflammatory syndrome in human exocrine pancreas, featured by repetitive pancreatic inflammation that usually results in irreversible pancreatic tissue damages such as pancreatic fibrosis, chronic pain, exocrine and endocrine pancreatic insufficiency, with an even higher risk of pancreatic cancer and reduced life expectancy. ${ }^{1,2}$ The annual CP incidence was reported to be near 10 per 100,000 individuals worldwide, and its prevalence was predicted to be over 120 per 100,000 individuals. $^{3}$ In recent years, the CP incidence has been continuously rising, but there are still no curative treatments for CP patients. ${ }^{1}$ Major known risk factors for CP include chronic alcohol abuse,
Correspondence: Ying Huang Department of Emergency, Hunan Provincial People's Hospital (The Firs Affiliated Hospital of Hunan Normal University), 61 Jiefang Road, Changsha, Hunan, 410005, People's Republic of China

Tel +8613974858993

Email carmen_huang@I63.com 
tobacco smoking, genetic mutations, hypercalcaemia, autoimmune disorder or even pancreatic tumors. ${ }^{3}$ Also, the complex pathophysiology of $\mathrm{CP}$ consists of multiple processes, including acinar cell damage, acinar stress responses, pancreatic ducal dysfunction, inflammation persistence and even neuro-immune crosstalk. ${ }^{3}$ However, the molecular mechanisms underlying CP development remain poorly understood.

Arginine methylation, the addition of methyl groups onto protein arginine (Arg) residues in their guanidino groups, is an important protein post-translational modification widely associated with various biological processes. ${ }^{4-8}$ Catalyzed by protein arginine methyltransferases (PRMTs), arginine methylation could be in different forms, including monomethylarginine (MMA), asymmetrical dimethylarginine (ADMA), or symmetrical dimethylarginine (SDMA). ${ }^{4-6}$ The modification with methyl groups was supposed to modulate protein functions via increasing arginine residue bulkiness and hydrophobicity, which interferes with the interactions between modified proteins and other biomacromolecule partners, ${ }^{6,9}$ thus regulating target gene transcription, pre-mRNA processing and protein translation. ${ }^{5,6}$ Extensive research in the past decades revealed that arginine methylation was widely involved in multiple biological and pathogenic processes, such as cell cycle progression, ${ }^{10}$ development and organogenesis, ${ }^{11}$ cardiovascular and neuronal functions, ${ }^{12}$ and cancer pathogenesis as well. ${ }^{5,13-17}$ Moreover, arginine methylation was essentially involved in the development of multiple chronic inflammation, mediated by the modification of transcriptional factors and co-factors associated with inflammation responses. ${ }^{18}$ However, the involvements of arginine methylation in CP pathogenesis remain largely unexplored.

Benefiting from the rapid technical progresses, proteomics based on mass spectrometry has been successfully applied for the characterization of protein arginine methylation associated with various biological and pathogenic processes in different species., ${ }^{9,13,19-28}$ Immunoaffinity purification, capable of efficiently enriching methylated arginine-containing peptides using antibodies specifically recognizing MMA, ADMA or SDMA, has been widely used for proteomic analysis of protein arginine methylation in the past years. ${ }^{9,13,23,26-28}$ For instance, 314 ADMA sites in 155 proteins have recently been identified by a comprehensive analysis of arginine methylation in colorectal cancer (CRC) tissues, which suggested that type I PRMTs catalyzing ADMA formation might be potential targets for developing new therapeutic drugs for CRC patients. $^{23}$ Moreover, the ADMA-containing protein profile in pancreatic cancer cells was also characterized by a combination of immunoaffinity purification and mass spectrometry. ${ }^{13}$ It is reasonable to predict that the largescale proteomic characterization of ADMA or SDMAcontaining proteins by immunoaffinity or other purification strategies might greatly deepen our understanding of disease pathogenic mechanisms mediated by arginine methylation.

In the present study, we performed a proteome-wide identification of ADMA-containing proteins as well as their modification sites in human CP tissues by coupling immunoaffinity enrichment and tandem mass spectrometry, which disclosed for the first time the association of protein arginine methylation with chronic inflammation in pancreas, and provided a basis for elucidating the roles of PRMTs and arginine methylation in CP pathogenesis.

\section{Materials and Methods Tissue Specimen Collection}

The treatment and management of chronic pancreatitis strictly follow the Chinese management guidelines for chronic pancreatitis. ${ }^{29}$ Surgical indications are as follows: patients with ineffective conservative and endoscopic treatment, intractable abdominal pain, severe local complications or highly suspected malignant transformation. Inclusion criteria are as follows: $\geq 18$ years old, diagnosed with CP by pathology and underwent surgical treatment. And exclusion criteria are as follows: history of pancreatic surgery, other chronic inflammatory and active malignancy. Totally 36 patients who were diagnosed with $\mathrm{CP}$ and underwent surgical treatment at the Department of Gastroenterology, Hunan Provincial People's Hospital (Changsha, China) between 6th March 2016 and 9th September 2020 were enrolled in this study. The median age of CP patients was 56 (rang from 48 to 68) years, and 20 patients were male. Three male CP cases were applied for proteomic analysis of ADMA-containing peptides, 4 $\mathrm{CP}$ cases for western-blotting verification of HNRNPA3 modification level, and 29 cases for analysis of the correlation of HNRNPA3 modification level with peripheral serum inflammatory factor levels. For these CP patients, $3 \mathrm{~mL}$ of venous blood was reserved, while pancreatic tissue was collected, and serum was centrifuged and stored at $-80^{\circ} \mathrm{C}$ for detection of inflammatory factors.

Seven other patients who were diagnosed with cholangiocarcinoma without pancreas involvements or patients 
with benign pancreatic tumors in the same hospital were included as the control group. Inclusion criterion was $\geq 18$ years old. And exclusion criteria include a history of pancreatic surgery or radiotherapy and chemotherapy. Among these patients, 3 male cases were applied as the control for proteomic analysis of ADMA-containing peptides, and the other 4 cases were used as the control for HNRNPA3 modification-level verification by the combination of immunoprecipitation and Western blot.

Pancreatic tissues surgically collected from the $\mathrm{CP}$ patients and the control group were immediately rinsed with PBS solution, sliced into small pieces $\left(1 \mathrm{~cm}^{3}\right)$, and stored in liquid nitrogen for following analyses. This research was approved by the Ethical Committee of Hunan Provincial People's Hospital (The First Affiliated Hospital of Hunan Normal University) and strictly following the Declaration of Helsinki. Written informed consents were signed by each participant before the surgical treatment.

\section{Total Protein Extraction}

Total protein samples were extracted from frozen human pancreatic tissues using the SDT buffer (4\% (w/v) SDS, $100 \mathrm{mM}$ Tris/HCl $\mathrm{pH}$ 7.6, $0.1 \mathrm{M}$ DTT) as previously introduced. $^{30}$ The concentration of pancreatic proteins was determined by the BCA method. The quality and homogeneity of total protein samples were assessed using SDS-PAGE and Coomassie brilliant blue staining. A preliminary LC-MS/MS (liquid chromatography-tandem mass spectrometry) analysis was performed to detect protein numbers in each sample. Subsequently, protein samples were mixed with the same volume of UA buffer ( 8 $\mathrm{M}$ urea, $150 \mathrm{mM}$ Tris- $\mathrm{HCl}, \mathrm{pH} 8.0$ ), condensed by filtering through ultrafiltration tubes, and the supernatant collected after centrifugation at $14,000 \mathrm{~g}$ for $20 \mathrm{~min}$ was quantized and subjected to tryptic digestion.

\section{In-Solution Tryptic Digestion}

Total proteins $(>1.0 \mathrm{mg})$ extracted from human pancreatic tissues were mixed with a DTT solution (final concentration: $10 \mathrm{mM}$ ) and incubated at $37^{\circ} \mathrm{C}$ for $1.5 \mathrm{~h}$ on an electronic shaker $(600 \mathrm{rpm})$. After being cooled at room temperature, protein samples were incubated with IAM (iodoacetamide; $50 \mathrm{mM}$ ) for $25 \mathrm{~min}$ in dark, diluted with 6 volumes of $50 \mathrm{mM}$ Tris $\mathrm{HCl}(\mathrm{pH} \mathrm{8.0)}$ ), and digested by incubation at $37^{\circ} \mathrm{C}$ for $17.5 \mathrm{~h}$ with trypsin (Sigma-Aldrich) under the protein/trypsin ratio (w/w) of 50:1. Finally, the $\mathrm{pH}$ value of peptide solutions were adjusted to $<3.0$ by adding 10\% TFA solution (final concentration: $0.1 \%$ ). After being desalted with a C18 SPE (solid-phase extraction) cartridge column, peptide solution was lyophilized for ADMA-containing peptide enrichment.

\section{Immunoaffinity Purification}

The ADMA-containing peptides were enriched by immunoaffinity purification using the PTMScan Asymmetric DiMethyl Arginine Kit (\#13474; Cell Signaling Technology) according to the manufacturer's instructions with minor modifications. Briefly, peptide samples obtained from tryptic digestion were first resuspended in a $1.5 \mathrm{~mL}$ pre-cooled IAP solution on ice, incubated with ADMA-R immunoaffinity beads at $4{ }^{\circ} \mathrm{C}$ for $2 \mathrm{~h}$, and centrifuged at $2000 \mathrm{~g}$ for 35 sec, and the resultant supernatants were discarded. Subsequently, ADMA-R immunoaffinity beads were washed with IAP buffer for three times, washed with precooled distilled water for three times, incubated twice with $40 \mu \mathrm{L} 0.15 \%$ TFA solution for $12 \mathrm{~min}$, and then the supernatants containing dimethylated peptides were desalted using a C18 STAGE Tips column and lyophilized for identification by LC-MS/MS.

\section{Mass Spectrometry}

The identification of ADMA-containing peptides by LCMS/MS method was performed as previously described, ${ }^{13}$ using the Thermo Scientific Q Exactive MS system, which is coupled online to an EASY-nLC 1000 liquid chromatography. Briefly, the enriched ADMA-containing peptides were first dissolved in 5\% acetonitrile/0.1\% TFA solution, loaded onto Acclaim PepMap100 loading column (Thermo Scientific; $100 \mu \mathrm{m} \times 20 \mathrm{~mm}$, nanoViper C18) via the autosampler, separated by an EASY column $(75 \mu \mathrm{m} \times$ $250 \mathrm{~mm}$ ) which is filled with C18-A2 particles $(3 \mu \mathrm{m})$. The mobile phases buffer A and B used for LC were $0.1 \%$ FA and $84 \%$ acetonitrile containing $0.1 \%$ FA, respectively, with a flow rate of $300 \mathrm{~nL} / \mathrm{min}$. The non-linear gradients for LC were set as follows: 5-8\% buffer B for $12 \mathrm{~min}, 8-$ $20 \%$ buffer B for $55 \mathrm{~min}, 20-30 \%$ buffer B for $8 \mathrm{~min}, 30-$ $100 \%$ buffer B for $4 \mathrm{~min}$, and 100\% buffer B for $10 \mathrm{~min}$.

The mass spectrometric identification of ADMAcontaining peptides was subsequently carried out on the Q Exactive MS system using the following settings: detection mode: positive ion; precursor ion scale: 300-1800 m/ z; MS resolution: at 70,000 at $200 \mathrm{~m} / \mathrm{z}$; AGC (Automatic gain control) target: $10^{6}$ ions; maximum injection time: 50 ms; dynamic exclusion: $60.0 \mathrm{sec}$. The fragmentation via HCD (higher-energy C-trap dissociation) with 
a normalized collision energy of $30 \mathrm{eV}$ was performed on 20 precursor ions obtained during each full scan, under the MS2 activation type of HCD. The isolation window was set to $2 \mathrm{~m} / \mathrm{z}$, MS2 resolution was 17,500 at $200 \mathrm{~m} / \mathrm{z}$ and the underfill was $0.1 \%$. The raw data obtained by LC-MS /MS were submitted to the ProteomeXchange Consortium through the iProX partner repository (Dataset ID: PXD026106).

\section{Database Search}

Raw data files obtained from LC-MS/MS were subjected to searching against the UniProt Homo sapiens proteome database containing 20, 244 entries (released on Jan 30, 2018), using the MaxQuant 1.6.0.16 software. Trypsin was selected as the protein digestion enzyme, with a maximum allowable mis-cleavage number of 2 . The minimum peptide length and the maximum peptide mass were set to 7 amino acids and $4600 \mathrm{Da}$, respectively, using $20 \mathrm{ppm}$ as the tolerance for precursor or fragment ions. Meanwhile, the fixed modification was cysteine carbamidomethylation, and variable modifications were set to arginine dimethylation $(28.0313 \mathrm{Da})$, methionine oxidation and N-terminal acetylation. The FDR (false discovery rate) of $<0.01$ was applied as criteria for reliable peptide and protein identification, and peptide modification was accepted when Andromeda score $>40$ and localization probability $>0.50$.

\section{HNRNPA3 Dimethylation Level}

The relative levels of asymmetric arginine dimethylation of HNRNPA3 proteins in human pancreatic tissues were detected by the immunoprecipitation using anti-ADMA antibodies (\#PTM-605RM; PTM BIO, Hangzhou, China), followed by Western blotting using antiHNRNPA3 antibodies (\#PA5-41961; Thermo Fisher Scientific). The immunoprecipitation procedure for collecting ADMA-containing proteins was carried out using the Dynabeads Protein A Immunoprecipitation Kit (\#10006D; Thermo Fisher Scientific) according to the manufacturer's instructions. Briefly, the anti-ADMA antibodies were first bound with the Dynabeads following the kit producer's instructions, which were used to mix with about $400 \mathrm{ul}$ total proteins extracted from human pancreatic tissues and incubated for 15 at room temperature with gentle rotation. After five washes with $200 \mu \mathrm{L}$ Washing Buffer, the ADMA-containing proteins were collected by incubating with about $30 \mu \mathrm{L}$ Elution Buffer, and used for Western blotting analysis using the antibodies against HNRNPA3.

\section{Inflammatory Factor Contents}

The serum inflammatory factor levels in the $\mathrm{CP}$ patients were determined by the ELISA (enzyme linked immunosorbent assay) method using commercially available experimental kits. Specifically, the levels of transforming growth factor beta (TGF- $\beta$ ) (\#CSB-E04725h), tumor necrosis factor alpha (TNF- $\alpha$ ) (\#CSB-E04740h) and interleukin-6 (IL-6) (\#CSB-E04638h) were detected strictly following the instructions of the manufacturer (CUSABIO, Wuhan, China).

\section{Bioinformatics}

The characteristics of amino acid sequences flanking ADMA sites were analyzed using the pLogo website. Functional categorization of ADMA-containing proteins based on GO (gene ontology) biological process, cellular component, molecular function and KEGG (Kyoto Encyclopedia of Genes and Genomes) pathways was performed using the DAVID (Database for Annotation, Visualization and Integrated Discovery) database. Differences in protein dimethylation levels were analyzed by the student $t$-test using the SPSS 20.0 software, and significant differences were defined by a $\mathrm{P}$ value of $<0.05$. The correlation of protein dimethylation level with inflammatory factor contents were assessed using the Pearson Correlation Coefficient.

\section{Results}

\section{Global Profiling of ADMA-Containing Proteins in CP Tissues}

For proteomic characterization of ADMA-containing proteins, the pancreatic tissues collected from $\mathrm{CP}$ patients were subjected to total protein extraction, tryptic digestion, immunoaffinity purification and mass spectrometry, using normal human pancreatic tissues as the control groups (Figure 1A). Three biological replicates were performed during the proteomic analysis (Figure 1B and C). Totally, 134 and 137 ADMA sites were identified in the control and CP groups, respectively (Figure 1B-D; Supplemental Table S1). Among them, 118 ADMA sites were identified in both the control and CP groups, while 16 and 19 ADMA sites were exclusively identified in control and CP groups, respectively (Figure 1D). These modification sites belong to 93 and 94 ADMA-containing proteins in the control and $\mathrm{CP}$ groups, respectively (Figure 1E; Supplemental Table S1). Totally 82 ADMA-proteins were characterized in both groups, while 11 and 12 
A

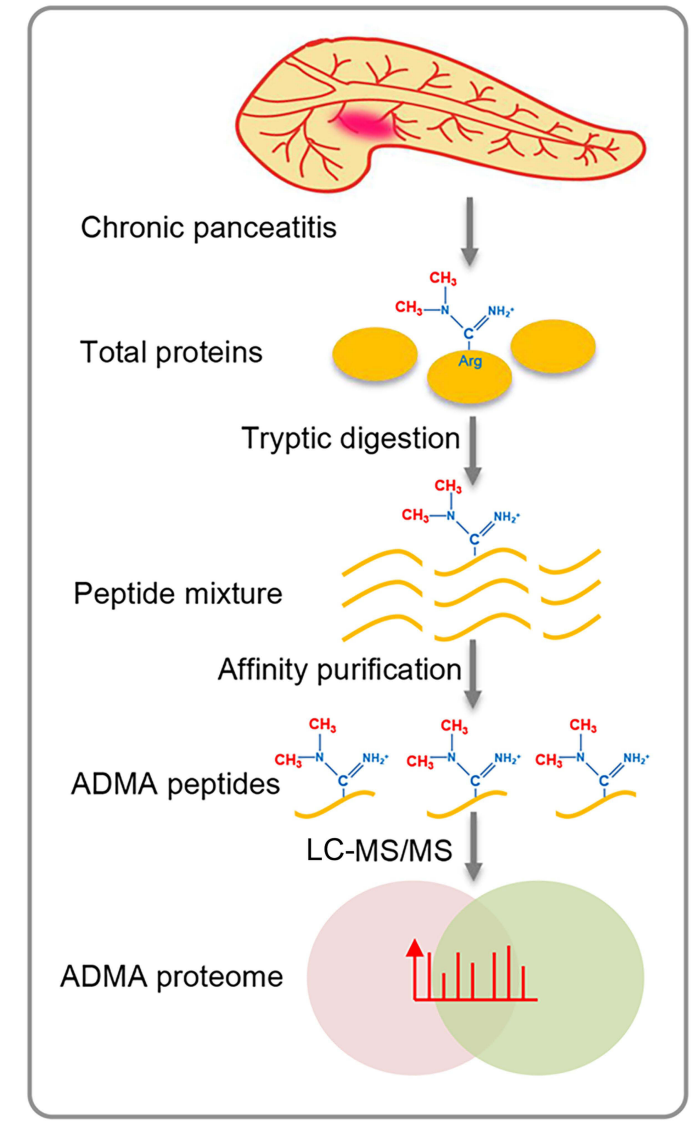

F

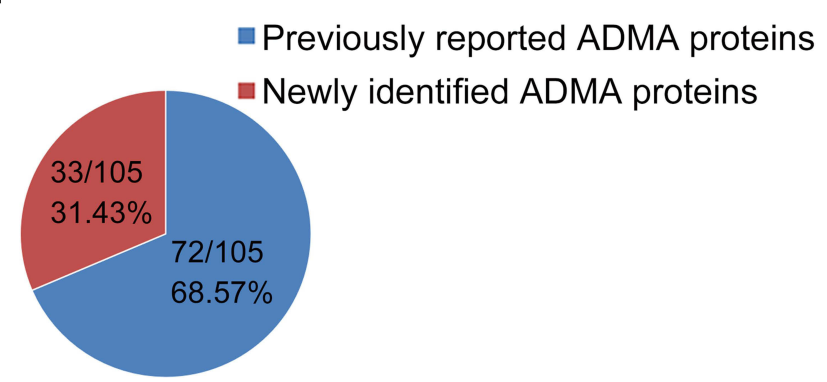

B

Total ADMA sites in Control

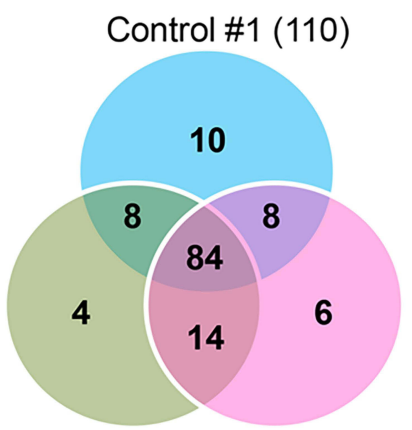

Control \#2

(110)

Control \#3

(112)

D

ADMA sites (153)

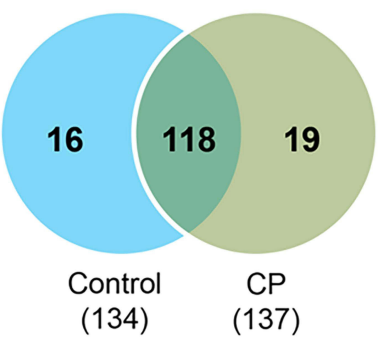

E
Total ADMA sites in CP

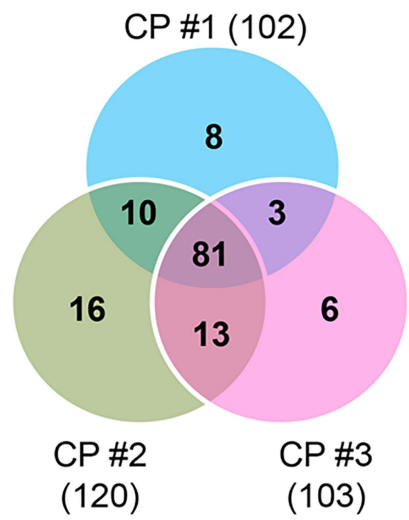

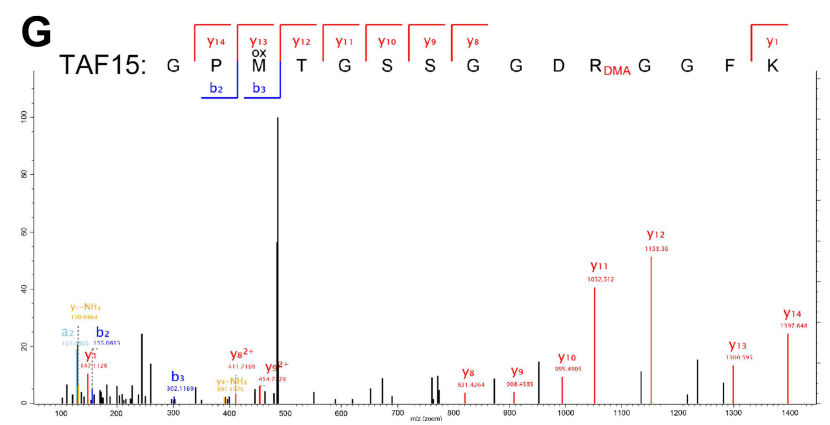

Figure I Proteomic characterization of ADMA-containing proteins in CP tissues. (A) Schematic illustration of proteomic procedures for characterizing ADMA-containing peptides in CP patients. Total proteins extracted from human CP tissues were subjected to tryptic digestion and affinity purification, and ADMA-containing peptides were finally identified by LC-MS/MS. (B and C) Numbers of ADMA-containing peptides identified in the control and CP tissues. Numbers of ADMA-containing peptides in three biological replicates were shown in Venn diagrams. (D) Total numbers of ADMA-containing peptides identified in the control and CP tissues. (E) Total numbers of ADMA-containing proteins identified in the control and CP tissues. (F) The percentages of newly identified and previously reported ADMA-containing proteins. Specific numbers of ADMA-containing protein numbers and percentages were presented on the pie chart. (G) Asymmetric dimethylation of TAFI 5 protein at Arg206 identified by mass spectrometry.

Abbreviations: ADMA, asymmetric dimethylarginine; MS, mass spectrometry; CP, chronic pancreatitis; TAFI5, TATA-binding protein associated factor I5.

proteins were identified exclusively in the control and $\mathrm{CP}$ groups, respectively (Figure 1E). Moreover, we showed that $68.57 \%(72 / 105)$ ADMA-containing proteins were identified in previous reports, showing the reliability of our proteomic dataset (Figure 1F). For instance, the ADMA at Arg206 in TAF15 (TATA-binding protein associated factor 15), which was previously identified in Hela cells, $^{31}$ was also characterized here in CP tissues
(Figure 1G). Also, 33 newly identified ADMAcontaining proteins were reported here in $\mathrm{CP}$ tissues (Figure 1F).

\section{Enrichment of Glycine Residue Near ADMA Sites in CP Tissues}

It has been shown by previous consensus sequences analysis that the flanking sequences of ADMA sites are 
characterized by significant enrichments of proline and glycine residues. ${ }^{9,13,26}$ Here in this study, we also analyzed the characteristics of amino acid residues flanking ADMA sites in human CP tissues using the pLogo software (Figure 2). In consistency with previous findings, we showed that glycine residue was preferentially enriched in several positions flanking the ADMA sites, especially at the positions $+1,+12$ and +15 (Figure 2A-C). Moreover, the proline residue was found to be overrepresented at the position -1 of ADMA sites, especially in those ADMA-containing peptides identified in the control group (Figure 2B). In addition, the glycine and proline residues were also shown to be enriched to different degrees in the flanking sequences of ADMA sites at multiple other positions (Figure 2A-C). These results obtained in $\mathrm{CP}$ tissue analysis further supported the roles of glycine and proline residues in facilitating ADMA formation.

\section{Functional Categorization of} ADMA-Containing Proteins in CP Tissues

To explore the potential pathogenic effects of asymmetric arginine dimethylation on $\mathrm{CP}$ development, we then performed a functional categorization of these ADMAcontaining proteins identified in human $\mathrm{CP}$ tissues based on GO (gene ontology) terms. In terms of biological processes $(\mathrm{BP})$, we found that ADMA-containing proteins in $\mathrm{CP}$ tissues were significantly enriched in RNA metabolism, RNA splicing, mRNA metabolism and splicing, intracellular transport, forebrain development, and mRNA stability regulation processes (Figure 3A). Besides these RNA-related processes, we showed that ADMA-containing proteins in the control group were also enriched in the transcytosis process, which was not observed in the CP group (Figure 3A). Moreover, ADMAcontaining proteins in $\mathrm{CP}$ tissues were distributed into various subcellular locations, including the nuclear lumen, cytoskeleton, nucleoplasm, nucleolus, spliceosome, nuclear body, nuclear speck and endocytic vesicle, and similar subcellular distributions were observed in the control group (Figure 3B). In addition, we showed that ADMA-containing proteins identified in $\mathrm{CP}$ tissues were mainly associated with DNA and RNA binding, cytoskeletal protein (actin) binding, SH3 (Src-homology 3) domain binding, translation factor activity and mRNA binding, in terms of molecular function (Figure 3C). These analyses indicate that the regulation of RNA processing like mRNA splicing by asymmetric arginine dimethylation might perform mediating roles in $\mathrm{CP}$ pathogenesis.

\section{Enrichment of ADMA-Containing Proteins in the Spliceosome Pathway}

As described above, ADMA-containing proteins in $\mathrm{CP}$ tissues were greatly enriched in the mRNA processing and splicing processes. We subsequently analyzed the association of modified proteins with the KEGG (Kyoto Encyclopedia of Genes) pathways, and found that ADMAcontaining proteins identified in $\mathrm{CP}$ tissues were significantly enriched in the spliceosome pathway (Figure 4). Among them, the Arg4165 of SF3B2 (Splicing factor 3B subunit 2; ID: Q13435), the Arg164 and Arg248 in HNRNPA1 (Heterogeneous nuclear ribonucleoprotein A1; ID: P09651), the Arg41 and Arg46 in HNRNPA3 (Heterogeneous nuclear ribonucleoprotein A3; ID: P51991), as well as the Arg241 and Arg958 in HNRNPU (Heterogeneous nuclear ribonucleoprotein U; ID: Q00839), were all identified as ADMA-containing sites in our proteomic analysis of $\mathrm{CP}$ tissues (Figure 4; Supplemental Table S1). These four proteins are essential members of the U2 and other protein complexes in the spliceosome, which further indicates that the modulation of mRNA splicing by asymmetric arginine dimethylation might perform critical roles in $\mathrm{CP}$ development and progression.

\section{Elevated HNRNPA3 Dimethylation Correlated with CP Progression}

To further support the involvements of ADMA in CP development, we then validated the asymmetric arginine dimethylation of HNRNPA3 protein in CP tissues, through the combination of immunoprecipitation using antiADMA antibodies and Western blot analysis targeting HNRNPA3. Compared with the control group consisting of four volunteers, we found that the HNRNPA3 asymmetric arginine dimethylation levels in the pancreatic tissues from four CP patients were greatly elevated, which is consistent with the above-mentioned proteomic analysis results (Figure 5A and $\mathrm{B}$ ). For more information on the potential roles of HNRNPA3 dimethylation in CP pathogenesis, we further analyzed the HNRNPA3 asymmetric arginine dimethylation levels among another 29 CP patients as introduced above, as well as the contents of major inflammatory factor contents in their serums 
A

ADMA sites in the Control groups

FG - unknown; BG - Human protein

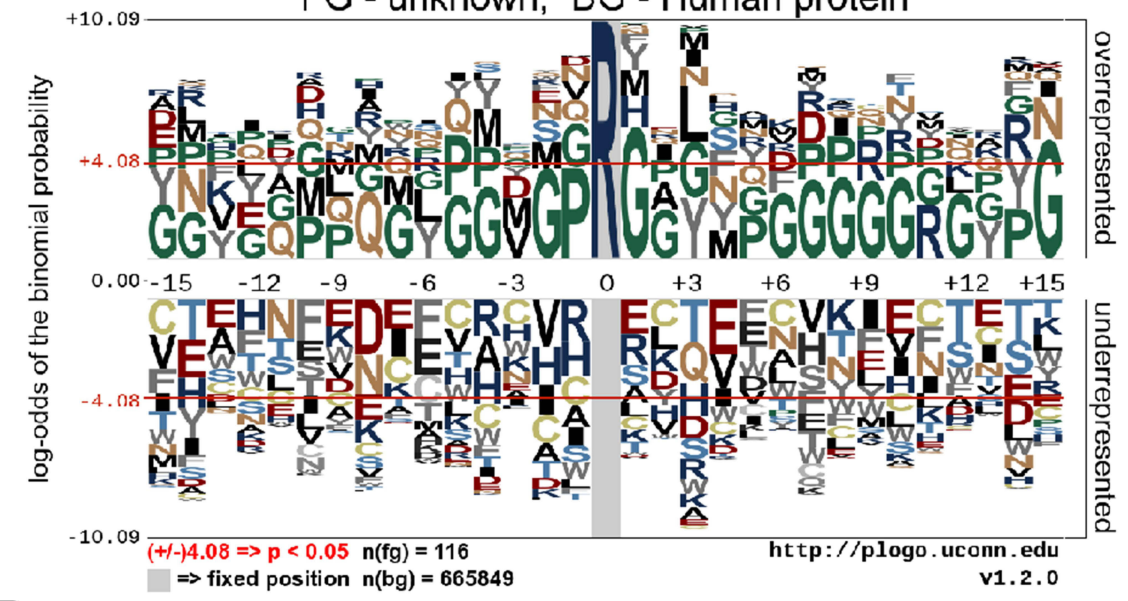

B

ADMA sites in the CP groups

FG - unknown; BG - Human protein

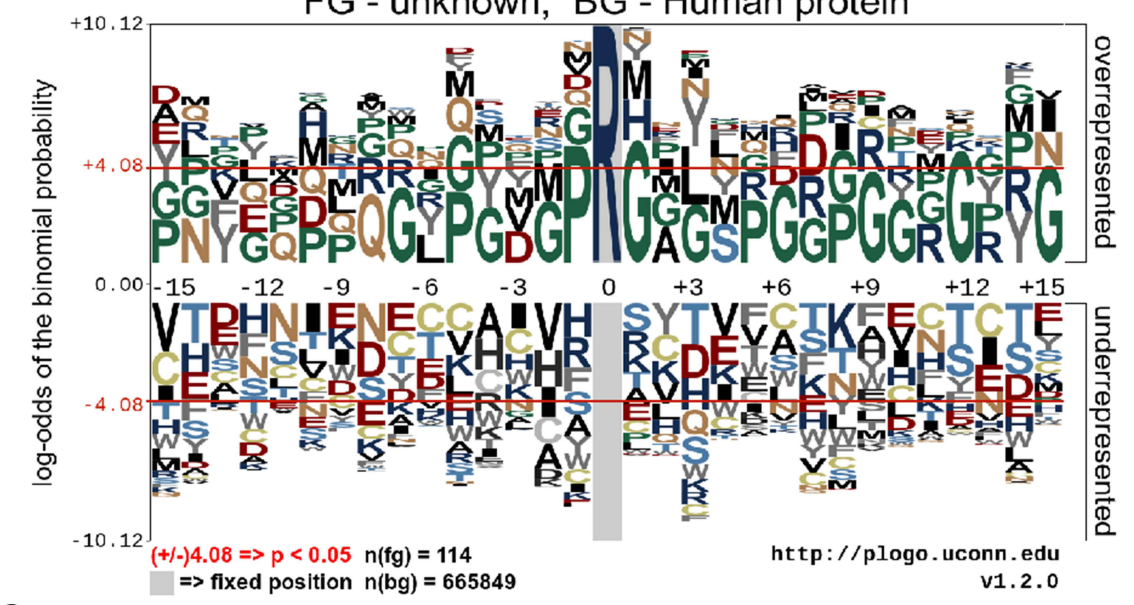

C

Total ADMA sites

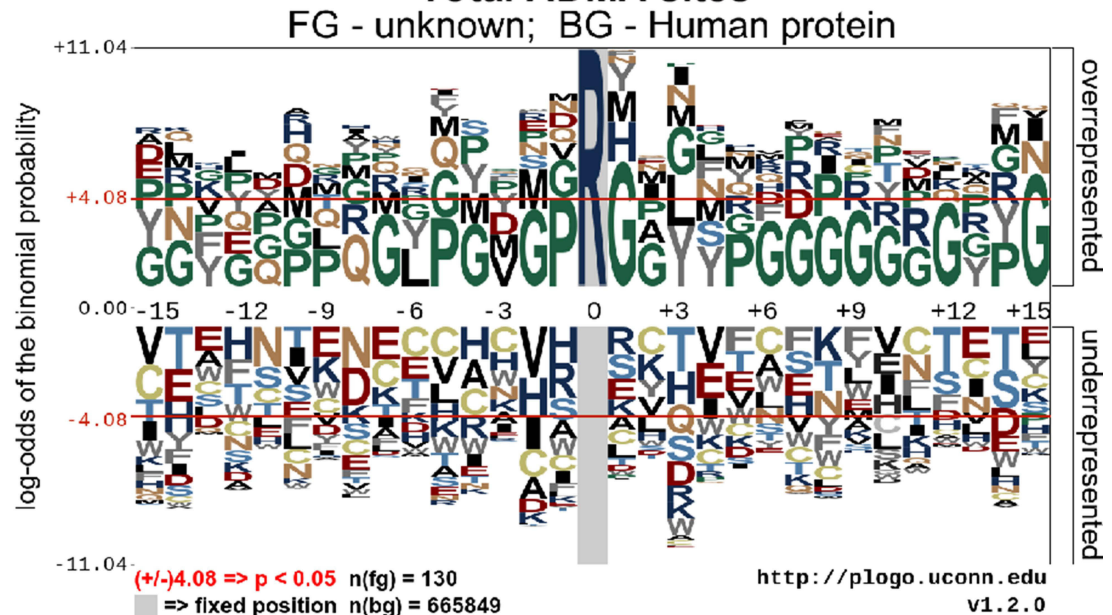

Figure 2 Characteristics of amino acid sequences flanking ADMA sites in CP tissues. The amino acid sequences of ADMA-containing peptides identified by proteomics in $\mathrm{CP}$ tissues, each of which contains amino acid residues from position -15 to position +15 , were subjected to consensus sequences analysis by the pLogo software (http:// plogo.uconn.edu). ADMA-containing peptides in the control group (A), CP group (B) and total ADMA-containing peptides (C) were analyzed separately. The methylated arginine $(R)$ residues were highlighted in deep blue color, and a $P$ value of $<0.05$ was used as the threshold of significant enrichments in the flanking sequences.

Abbreviations: ADMA, asymmetric dimethylarginine; $C P$, chronic pancreatitis. 


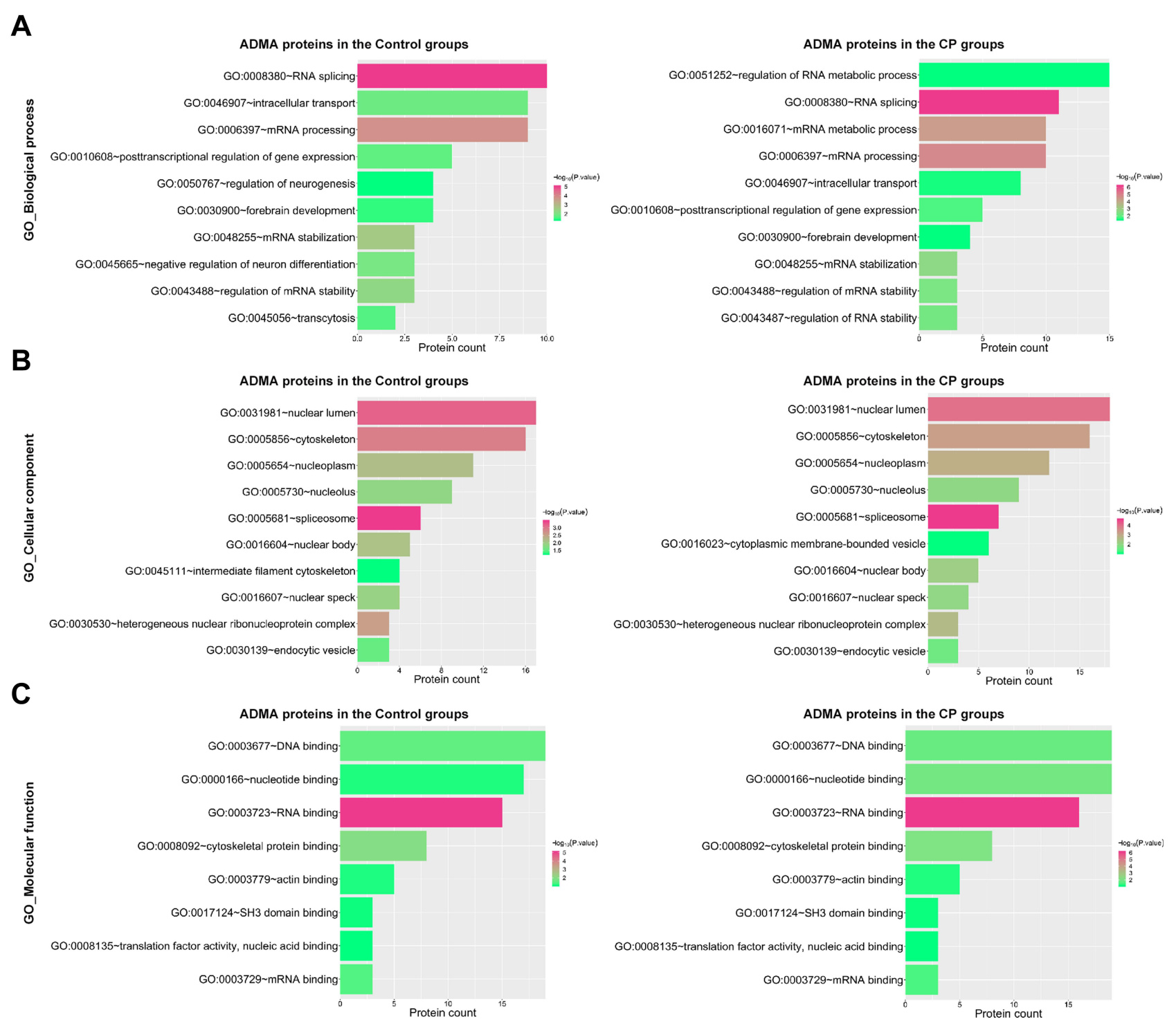

Figure 3 Functional clustering of ADMA-containing proteins in CP tissues. The enrichments of ADMA-containing proteins in gene ontology (GO) terms were analyzed using the DAVID websites, based on the biological process (A), cellular component (B) and molecular function (C). ADMA-containing proteins identified in the control group and the CP group were analyzed separately. The numbers of ADMA-containing proteins associated with each term were indicated in the $X$ axis. $A P$ value of $<0.05$ was used for definition of significant enrichment, which was shown in red or green bars based on the significance degrees.

Abbreviations: ADMA, asymmetric dimethylarginine; CP, chronic pancreatitis; GO, gene ontology.

(Figure 5C-E). We observed that the asymmetric arginine dimethylation levels of HNRNPA3 in CP patients exhibited significantly positive correlation with the contents of serum TGF- $\beta\left(\mathrm{R}^{2}=0.4764, P<0.01\right)$, TNF- $\alpha\left(\mathrm{R}^{2}=0.1608\right.$, $P<0.01)$ and IL-6 $\left(\mathrm{R}^{2}=0.1909, P<0.01\right)$ (Figure $5 \mathrm{C}-\mathrm{E}$ ). These results further indicated the possible functions of protein arginine dimethylation such as HNRNPA3 in CP initiation and progression.

\section{Discussion}

Chronic pancreatitis (CP) is a common inflammation syndrome in the human digestive system associated with continuously increasing incidence and high risk of progressing into pancreatic cancer, and recently, there are still no curative treatments in clinical management. ${ }^{1,2}$ However, the molecular mechanisms mediating CP development and progression still remain poorly understood. The methylation of protein arginine residues was previously shown to mediate chronic inflammation processes, ${ }^{18}$ but its involvements in CP development have not been explored. In this study, we performed the first proteome-wide characterization of ADMA-containing proteins and their modification sites in inflammatory tissues collected from $\mathrm{CP}$ patients by immunoaffinity 


\section{ADMA proteins associated with the spliceosome pathway}

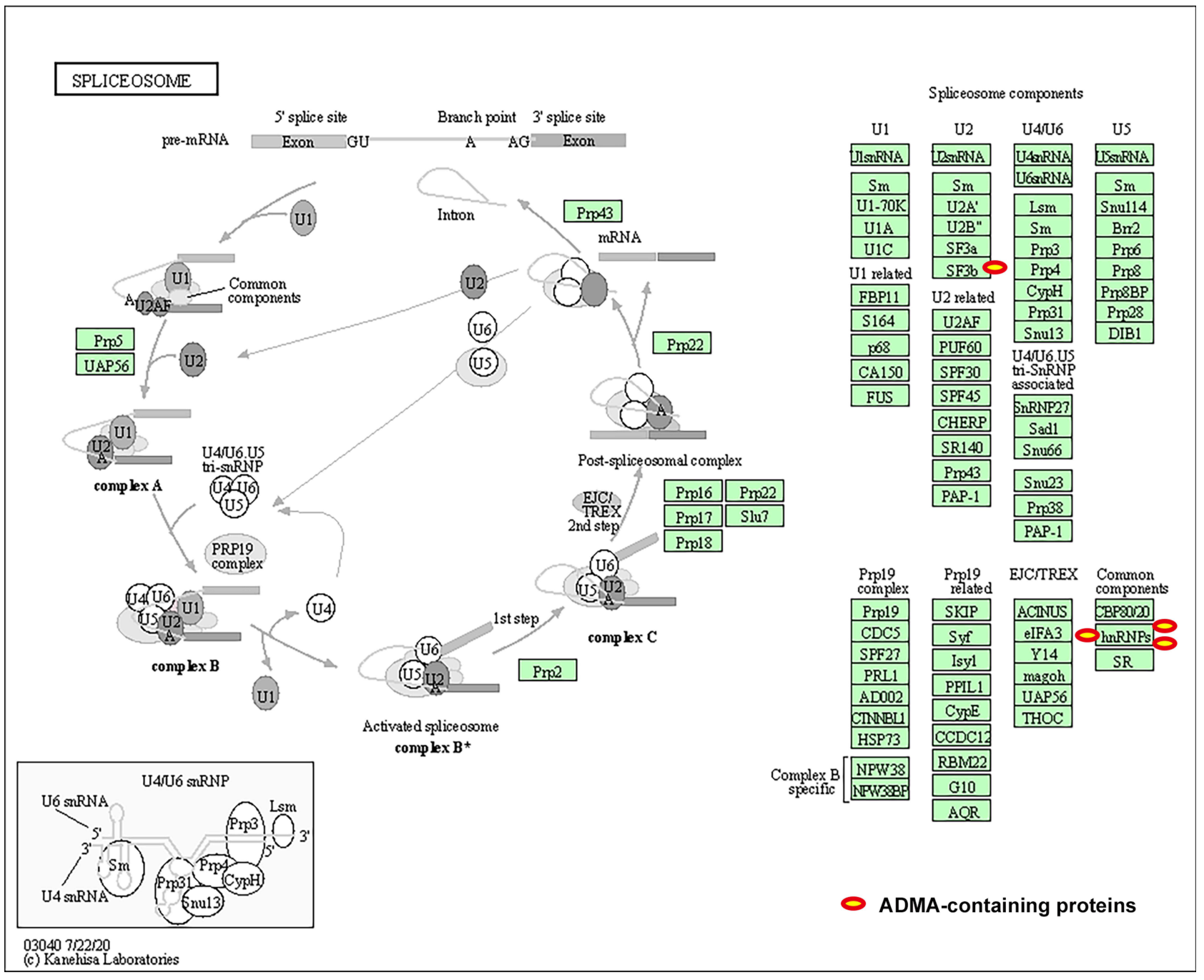

Figure 4 ADMA-containing proteins associated with spliceosome in CP tissues. The enrichment of ADMA-containing proteins in the KEGG pathways were analyzed using the DAVID website (https://david.ncifcrf.gov/home.jsp). The spliceosome pathway diagrams were modified from the KEGG database. ADMA-containing proteins involved in the spliceosome pathways were marked with red circles.

Abbreviations: ADMA, asymmetric dimethylarginine; KEGG, Kyoto Encyclopedia of Genes.

purification and mass spectrometry, to address the potential roles of arginine methylation in $\mathrm{CP}$ pathogenesis. ADMA-containing proteins in $\mathrm{CP}$ tissues were found to be significantly enriched in mRNA splicing and other RNA splicing processes. Importantly, multiple key protein members of the spliceosome pathway such as HNRNPA3 were identified as ADMA-containing proteins in $\mathrm{CP}$ tissues. Moreover, HNRNPA3 dimethylation levels were greatly elevated in CP tissues and showed positive correlation with inflammatory factors. These results suggested the possible functions of protein arginine methylation in the CP pathogenesis.

PRMT-catalyzed arginine methylation in histone or non-histone proteins plays essential roles in human disease development and progression, mediated by epigenetic regulation of gene expression and signaling pathways. $5,15,17$ Moreover, PRMTs have also been reported to mediate inflammatory responses in multiple human systems, by acting as coactivators of inflammation-related factors or catalyzing arginine methylation in inflammatory factors. ${ }^{18}$ For instance, PRMT4 (protein arginine methyltransferase 4), also known as CARM1 (coactivator-associated arginine methyltransferase 1 ), could regulate the expression of inflammatory genes by enhancing the recruitment of NF-kB (nuclear factor-kappaB) to chromatin. ${ }^{32}$ Also, PRMT1 regulates the NF-kB-dependent expression of inflammatory factors through methylating Arg-3 in histone $\mathrm{H} 4$ or methylating other NF-kB transcriptional 
A

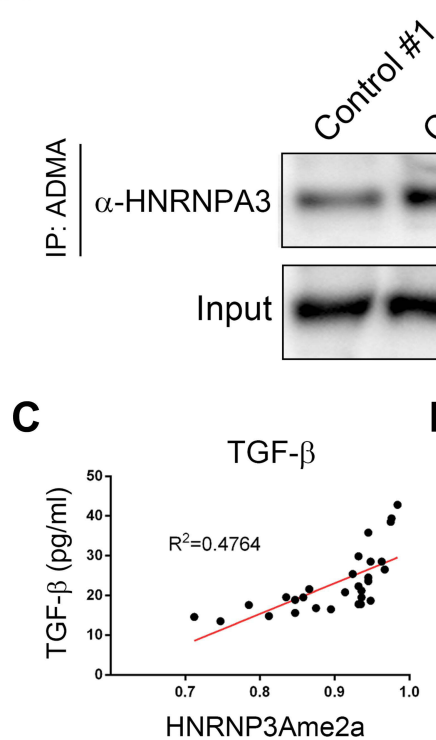

B
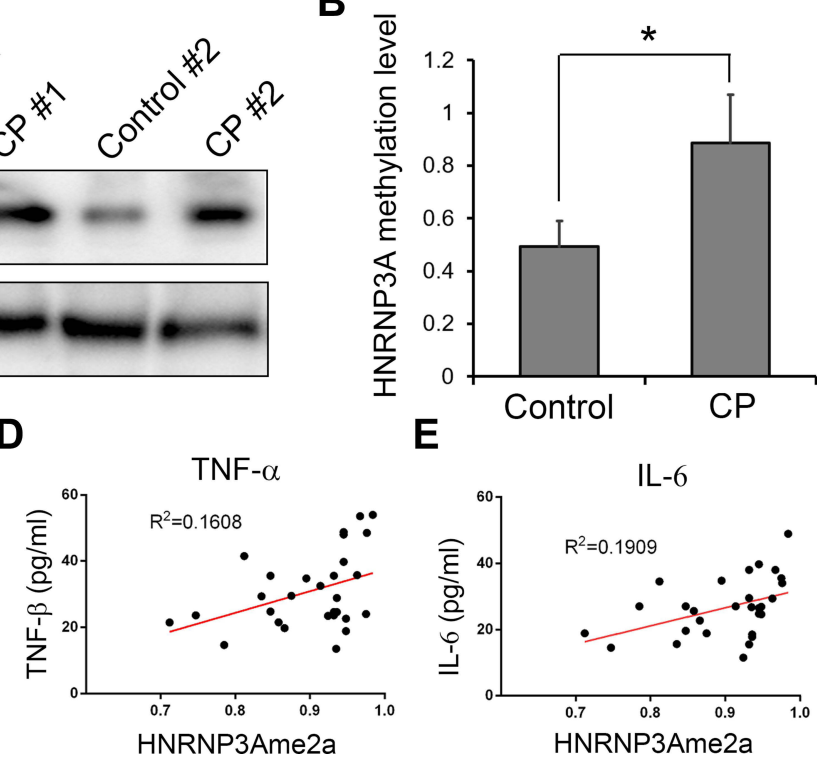

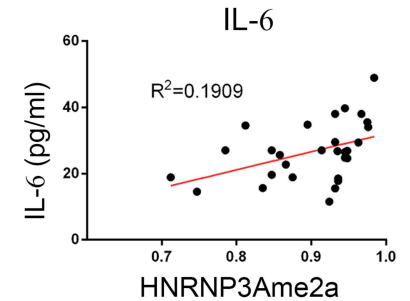

Figure 5 HNRNPA3 dimethylation and its correlation with inflammatory factors. (A) Greatly increased asymmetric arginine dimethylation of HNRNPA3 proteins in CP tissues. Protein asymmetric arginine dimethylation were quantitatively assessed by immunoprecipitation using antibodies targeting ADMA, followed by Western blot with the antibodies specifically recognizing HNRNPA3 (B). Relative HNRNPA3 dimethylation levels among four CP patients were compared with the control group containing four other cholangiocarcinoma patients. (C, Dand E) The positive correlation of HNRNPA3 asymmetric arginine dimethylation levels with the major inflammatory factors in serum of CP patients. Serum TGF-b, TNF-a and IL-6 levels in CP patients were analyzed via ELISA method. $* P<0.05$.

Abbreviations: HNRNPA3, heterogeneous nuclear ribonucleoprotein A3; ADMA, asymmetric dimethylarginine; CP, chronic pancreatitis; TGF- $\beta$, transforming growth factor- $\beta$; TNF- $\alpha$, tumor necrosis factor alpha; IL-6, interleukin-6.

coactivators such as Poly(ADP-ribose) polymerase 1 (PARP1) and p160. ${ }^{18}$ Recently, PRMT1-catalyzed methylation of Smad6 (small mothers against decapentaplegic homolog 6) protein, which is a key member of TGF- $\beta$ induced anti-inflammatory signaling, was also shown to repress periodontal inflammation by regulating NF- $\mathrm{KB}$ activation. ${ }^{33}$ However, little is known about the association of PRMTs and arginine methylation with CP development. In this study, we showed that a large set of functional proteins underwent asymmetric arginine dimethylation in CP tissues, which suggested the roles of protein arginine methylation in regulating inflammation progression during $\mathrm{CP}$ pathogenesis.

As introduced above, sustained inflammation in $\mathrm{CP}$ has been established as one major causative factor for the development of pancreatic cancer. ${ }^{1,2}$ The predisposition to pancreatic cancer in $\mathrm{CP}$ patients was proposed to be partially mediated by digestive enzyme-secreting acinar cells, which could undergo ductal metaplasia under the inflammatory environment. ${ }^{34}$ However, the molecular mechanisms driving their malignant transformation after long-term pancreatitis progression still remain poorly elucidated. The pancreatic cancer cells possess proteomewide alterations of ADMA-containing protein profiles, which are associated with multiple biological processes and signaling pathways related to cancer development. ${ }^{13}$ In this study, we found that a large number of ADMAcontaining proteins in $\mathrm{CP}$ tissues were also characterized in the previous proteomic analysis in pancreatic cancer cells, including the BCL9L (B-cell CLL/lymphoma 9-like protein), RUNX1 (Runt-related transcription factor 1), and TAF15 (TATA-binding protein-associated factor 15) (See Supplemental Table S1). Among them, RUNX1 was recently shown to regulate the proliferation, migration and invasiveness of pancreatic cancer cells via interacting with microRNAs or IncRNAs. ${ }^{35,36}$ Moreover, the activity of RUNX1 could also be regulated by PRMT1-induced arginine methylation, which is associated with hematopoiesis and leukemia development. ${ }^{37}$ Based on these observations, it is reasonable to speculate that arginine methylation might mediate the progression of $\mathrm{CP}$ into pancreatic cancer.

The progression of inflammatory diseases is regulated by the alternative mRNA splicing of functional genes. ${ }^{38,39}$ Moreover, the pre-mRNA splicing defects were also established as risk factors for CP development. ${ }^{40}$ However, the mechanisms modulating mRNA splicing associated with CP pathogenesis are still largely unknown. Protein arginine methylation exerts various biological and pathogenic effects through influencing 
mRNA splicing, which is mediated by the methylation of key protein members or co-factors of the spliceosome complexes. $^{5,41}$ In this study, we found that ADMAcontaining proteins in CP tissues were significantly enriched in the spliceosome pathway, including several key spliceosome components, such as SF3B2, HNRNPA1, HNRNPA3 and HNRNPU. Among them, HNRNPA3 regulates RNA trafficking and processing during multiple processes, such as neural development and tumorigenesis, $^{42-45}$ which was also identified as one quantitative trait loci for murine pancreatitis development. ${ }^{43}$ In this report, we validated the significantly elevated HNRNPA3 dimethylation in CP tissues, which was correlated with major inflammatory factors, suggesting the possible involvement of arginine methylation-regulated RNA splicing in CP pathogenesis. Of note, the relationship of ADMA-proteins with inflammatory responses could be further addressed by comparing AMDA-containing proteins between the $\mathrm{CP}$ and other pancreatitis subtypes such as obstructive pancreatitis. Moreover, RNA splicing is also involved in other processes associated with $\mathrm{CP}$ progression such as autophagy and fibrosis, ${ }^{46}$ and the roles of ADMA in regulating these processes during $\mathrm{CP}$ development deserve further investigations.

It should be noted that this study is limited by the lack of functional investigations of ADMA-containing proteins. The pathogenic roles of protein arginine methylation in $\mathrm{CP}$ development should be further validated using animal models, which require the preparation of antibodies specifically targeting dimethylated peptides and the establishment of genetically modified animal model with point mutation of the dimethylated residues. Secondly, there are a limited number of CP patients who meet the surgical indications, which makes it difficult to verify the correlation of other identified ADMA-containing proteins with $\mathrm{CP}$ progression using clinical samples. In summary, we displayed here the first characterization of ADMAcontaining protein profiles associated with $\mathrm{CP}$ pathogenesis, which provided a basis for elucidating the roles of protein arginine methylation in pancreatitis development, and might possibly facilitate the development of acute diagnosis and curative treatments for $\mathrm{CP}$ clinical management.

\section{Funding}

This study was funded by grants from China National Natural Science Fund (81600502), Clinical Guidance
Project of Hunan Provincial Science and Technology Department (2020SK50927, 2020SK50926), Natural Science Foundation of Hunan Province (2020JJ8086, 2019JJ40164) and Excellent youth fund of Hunan Education Department (19B352).

\section{Disclosure}

The authors declare that they have no competing interests.

\section{References}

1. Beyer G, Habtezion A, Werner J, Lerch MM, Mayerle J. Chronic pancreatitis. Lancet. 2020;396(10249):499-512. doi:10.1016/s01406736(20)31318-0

2. Yang D, Forsmark CE. Chronic pancreatitis. Curr Opin Gastroenterol. 2017;33(5):396-403. doi:10.1097/ MOG.0000000000000377

3. Kleeff J, Whitcomb DC, Shimosegawa T, et al. Chronic pancreatitis. Nat Rev Dis Primers. 2017;3(1):17060. doi:10.1038/nrdp.2017.60

4. Murn J, Shi Y. The winding path of protein methylation research: milestones and new frontiers. Nat Rev Mol Cell Biol. 2017;18(8):517. doi:10.1038/nrm.2017.35

5. Jarrold J, Davies CC. PRMTs and arginine methylation: cancer's best-kept secret? Trends Mol Med. 2019;25(11):993-1009. doi:10.1016/j.molmed.2019.05.007

6. Guccione E, Richard S. The regulation, functions and clinical relevance of arginine methylation. Nat Rev Mol Cell Biol. 2019;20 (10):642-657. doi:10.1038/s41580-019-0155-x

7. Blanc RS, Richard S. Arginine methylation: the coming of age. $\mathrm{Mol}$ Cell. 2017;65(1):8. doi:10.1016/j.molcel.2016.11.003

8. Bedford MT, Clarke SG. Protein arginine methylation in mammals: who, what, and why. Mol Cell. 2009;33(1):1-13. doi:10.1016/j. molcel.2008.12.013

9. Shishkova E, Zeng H, Liu F, et al. Global mapping of CARM1 substrates defines enzyme specificity and substrate recognition. Nat Commun. 2017;8:15571.

10. Raposo AE, Piller SC. Protein arginine methylation: an emerging regulator of the cell cycle. Cell Div. 2018;13(1):3. doi:10.1186/ s13008-018-0036-2

11. Jambhekar A, Dhall A, Shi Y. Roles and regulation of histone methylation in animal development. Nat Rev Mol Cell Biol. 2019;20(10):625-641. doi:10.1038/s41580-019-0151-1

12. Couto ESA, Wu CY-C, Citadin CT, et al. Protein arginine methyltransferases in cardiovascular and neuronal function. Mol Neurobiol. 2020;57(3):1716-1732. doi:10.1007/s12035-019-01850-z

13. Wei M, Tan C, Tang Z, et al. Proteome-wide alterations of asymmetric arginine dimethylation associated with pancreatic ductal adenocarcinoma pathogenesis. Front Cell Dev Biol. 2020;8:545934. doi:10.3389/fcell.2020.545934

14. Poulard C, Corbo L, Le Romancer M. Protein arginine methylation/ demethylation and cancer. Oncotarget. 2016;7(41):67532-67550. doi:10.18632/oncotarget. 11376

15. Carlson SM, Gozani O. Nonhistone Lysine methylation in the regulation of cancer pathways. Cold Spring Harb Perspect Med. 2016;6 (11):a026435. doi:10.1101/cshperspect.a026435

16. Yang Y, Bedford MT. Protein arginine methyltransferases and cancer. Nat Rev Cancer. 2013;13(1):37-50. doi:10.1038/nrc3409

17. Waldmann T, Schneider R. Targeting histone modifications-epigenetics in cancer. Curr Opin Cell Biol. 2013;25(2):184-189. doi:10.1016/j.ceb.2013.01.001

18. Kim JH, Yoo BC, Yang WS, et al. The role of protein arginine methyltransferases in inflammatory responses. Mediators Inflamm. 2016;2016:4028353. doi:10.1155/2016/4028353 
19. Musiani D, Bok J, Massignani E, et al. Proteomics profiling of arginine methylation defines PRMT5 substrate specificity. Sci Signal. 2019;12(575). doi:10.1126/scisignal.aat8388

20. Zeeshan M, Kaur I, Joy J, et al. Proteomic identification and analysis of arginine-methylated proteins of plasmodium falciparum at asexual blood stages. J Proteome Res. 2017;16(2):368-383. doi:10.1021/acs. jproteome.5b01052

21. Fisk JC, Li J, Wang $\mathrm{H}$, et al. Proteomic analysis reveals diverse classes of arginine methylproteins in mitochondria of trypanosomes. Mol Cell Proteom. 2013;12(2):302. doi:10.1074/mcp.M112.022533

22. Sylvestersen KB, Horn H, Jungmichel S, Jensen LJ, Nielsen ML. Proteomic analysis of arginine methylation sites in human cells reveals dynamic regulation during transcriptional arrest. Mol Cell Proteom. 2014;13(8):2072-2088. doi:10.1074/mcp.O113.032748

23. Lim Y, Lee JY, Ha SJ, et al. Proteome-wide identification of arginine methylation in colorectal cancer tissues from patients. Proteome Sci. 2020;18(1):6. doi:10.1186/s12953-020-00162-8

24. Radzisheuskaya A, Shliaha PV, Grinev V, et al. PRMT5 methylome profiling uncovers a direct link to splicing regulation in acute myeloid leukemia. Nat Struct Mol Biol. 2019;26(11):999-1012. doi:10.1038/ s41594-019-0313-z

25. Uhlmann T, Geoghegan VL, Thomas B, et al. A method for large-scale identification of protein arginine methylation. Mol Cell Proteomics. 2012;11(11):1489-1499. doi:10.1074/mcp.M112.020743

26. Guo A, Gu H, Zhou J, et al. Immunoaffinity enrichment and mass spectrometry analysis of protein methylation. Mol Cell Proteomics. 2014;13(1):372-387. doi:10.1074/mcp.O113.027870

27. Geoghegan V, Guo A, Trudgian D, Thomas B, Acuto O. Comprehensive identification of arginine methylation in primary $\mathrm{T}$ cells reveals regulatory roles in cell signalling. Nat Commun. 2015;6:6758.

28. Yakubu RR, Silmon de Monerri NC, Nieves E, Kim K, Weiss LM. Comparative monomethylarginine proteomics suggests that Protein Arginine Methyltransferase 1 (PRMT1) is a significant contributor to arginine monomethylation in toxoplasma gondii. Mol Cell Proteomics. 2017;16(4):567-580. doi:10.1074/mcp.M117.066951

29. Zou WB, Ru N, Wu H, et al. Guidelines for the diagnosis and treatment of chronic pancreatitis in China (2018 edition). Hepatobiliary Pancreat Dis Int. 2019;18(2):103-109. doi:10.1016/j. hbpd.2019.02.004

30. Tong Z, Kim MS, Pandey A, Espenshade PJ. Identification of candidate substrates for the Golgi Tul1 E3 ligase using quantitative diGly proteomics in yeast. Mol Cell Proteomics. 2014;13(11):2871-2882. doi:10.1074/mcp.M114.040774

31. Jobert L, Argentini M, Tora L. PRMT1 mediated methylation of TAF15 is required for its positive gene regulatory function. Exp Cell Res. 2009;315(7):1273-1286. doi:10.1016/j.yexcr.2008.12.008

32. Covic M, Hassa PO, Saccani S, et al. Arginine methyltransferase CARM1 is a promoter-specific regulator of NF-kappaB-dependent gene expression. EMBO J. 2005;24(1):85-96. doi:10.1038/sj. emboj. 7600500
33. Zhang T, Wu J, Ungvijanpunya N, et al. Smad6 methylation represses nfkappab activation and periodontal inflammation. J Dent Res. 2018;97(7):810-819. doi:10.1177/0022034518755688

34. Pinho AV, Chantrill L, Rooman I. Chronic pancreatitis: a path to pancreatic cancer. Cancer Lett. 2014;345(2):203-209. doi:10.1016/j. canlet.2013.08.015

35. Cheng Y, Yang H, Sun Y, et al. RUNX1 promote invasiveness in pancreatic ductal adenocarcinoma through regulating miR-93. Oncotarget. 2017;8(59):99567-99579. doi:10.18632/ oncotarget.20433

36. Liu S, Zhang J, Yin L, et al. The 1ncRNA RUNX1-IT1 regulates C-FOS transcription by interacting with RUNX1 in the process of pancreatic cancer proliferation, migration and invasion. Cell Death Dis. 2020;11(6):412. doi:10.1038/s41419-020-2617-7

37. Zhao X, Jankovic V, Gural A, et al. Methylation of RUNX1 by PRMT1 abrogates SIN3A binding and potentiates its transcriptional activity. Genes Dev. 2008;22(5):640-653. doi:10.1101/gad.1632608

38. Gordon ED, Simpson LJ, Rios CL, et al. Alternative splicing of interleukin-33 and type 2 inflammation in asthma. Proc Natl Acad Sci USA. 2016;113(31):8765-8770. doi:10.1073/pnas.1601914113

39. Liu H, Lorenzini PA, Zhang F, et al. Alternative splicing analysis in human monocytes and macrophages reveals MBNL1 as major regulator. Nucleic Acids Res. 2018;46(12):6069-6086. doi:10.1093/ nar/gky401

40. Beer S, Sahin-Toth M. Exonic variants affecting pre-mRNA splicing add to genetic burden in chronic pancreatitis. Gut. 2014;63 (5):860-861. doi:10.1136/gutjnl-2013-305981

41. Hu J, Yang $\mathrm{H}, \mathrm{Mu} \mathrm{J}$, et al. Nitric oxide regulates protein methylation during stress responses in plants. Mol Cell. 2017;67(4):702-710.e4. doi:10.1016/j.molcel.2017.06.031

42. Ma AS, Moran-Jones K, Shan J, et al. Heterogeneous nuclear ribonucleoprotein A3, a novel RNA trafficking response element-binding protein. J Biol Chem. 2002;277(20):18010-18020. doi:10.1074/jbc. M200050200

43. Asghari F, Fitzner B, Holzhuter S-A, et al. Identification of quantitative trait loci for murine autoimmune pancreatitis. J Med Genet. 2011;48(8):557-562. doi:10.1136/jmg.2011.089730

44. Mishra N, Reddy KS, Timilsina U, Gaur D, Gaur R. Human APOBEC3B interacts with the heterogenous nuclear ribonucleoprotein A3 in cancer cells. J Cell Biochem. 2018;119(8):6695-6703. doi:10.1002/jcb.26855

45. Ou MY, Ju XC, Cai YJ, et al. Heterogeneous nuclear ribonucleoprotein A3 controls mitotic progression of neural progenitors via interaction with cohesin. Development. 2020;147:dev185132. doi:10.1242/dev.185132

46. Quidville V, Alsafadi S, Goubar A, et al. Targeting the deregulated spliceosome core machinery in cancer cells triggers mTOR blockade and autophagy. Cancer Res. 2013;73(7):2247-2258. doi:10.1158/ 0008-5472.CAN-12-2501
Journal of Inflammation Research

\section{Publish your work in this journal}

The Journal of Inflammation Research is an international, peerreviewed open-access journal that welcomes laboratory and clinical findings on the molecular basis, cell biology and pharmacology of inflammation including original research, reviews, symposium reports, hypothesis formation and commentaries on: acute/chronic inflammation; mediators of inflammation; cellular processes; molecular mechanisms; pharmacology and novel anti-inflammatory drugs; clinical conditions involving inflammation. The manuscript management system is completely online and includes a very quick and fair peerreview system. Visit http://www.dovepress.com/testimonials.php to read real quotes from published authors. 